\title{
Lentivirus-mediated silencing of spindle and kinetochore-associated protein 1 inhibits the proliferation and invasion of neuronal glioblastoma cells
}

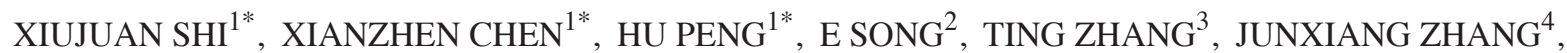 \\ JING LI ${ }^{4}$, HIMAYA SWA ${ }^{5}$, YONGXIN LI ${ }^{6}$, SEKWON KIM ${ }^{6}$, XIAOQING LIU ${ }^{1}$ and CHEN ZHANG ${ }^{1}$ \\ ${ }^{1}$ Shanghai Tenth People's Hospital, Tongji University School of Medicine, Shanghai 200072; \\ ${ }^{2}$ Lixiang Eye Hospital of Soochow University, Suzhou, Jiangsu 242000; ${ }^{3}$ Medical Technology College, \\ Zhejiang Chinese Medical University, Hangzhou, Zhejiang 310053; ${ }^{4}$ Xuancheng Central Hospital, Xuancheng, \\ Anhui 242000; ${ }^{5}$ Tongji Hospital Affiliated to Tongji University, School of Medicine, Tongji University, \\ Shanghai 200065, P.R. China; ${ }^{6}$ Specialized Graduate School Science and Technology Convergence, \\ Department of Marine Bio Convergence Science, Pukyong National University, Busan 608-737, Republic of Korea
}

Received February 28, 2014; Accepted November 12, 2014

DOI: $10.3892 / \mathrm{mmr} .2015 .3175$

\begin{abstract}
Spindle and kinetochore-associated protein 1 (SKA1) is an important component of the human kinetochore, which plays a key role in mitosis. The resent study was designed to investigate the role of SKA1 in human glioblastoma. The results of the present study demonstrated that SKA1 was expressed in human glioblastoma cells. In addition, the knockdown of SKA1 expression in the A172 and U251 human glioblastoma cell lines was accomplished using a lentivirus infection method. An MTT assay demonstrated that downregulation of SKA1 may inhibit cell proliferation, without affecting the cell cycle. Furthermore, knockdown of SKA1 expression resulted in reduced cell invasion. The results of the present study indicated that SKA1 may be a potential target protein for antiproliferative and anti-invasive therapeutic strategies of human glioblastoma.
\end{abstract}

\section{Introduction}

Spindle and kinetochore-associated protein 1 (SKA1) has been previously identified as a component of the human kinetochore (KT) (1-3). The core of the SKA1 complex is composed of

Correspondence to: Dr Chen Zhang or Dr Xiaoqing Liu, Shanghai Tenth People's Hospital, Tongji University School of Medicine, 301 Middle Yangchang Road, Shanghai 200072, P.R. China

E-mail: drchenzhang@126.com

E-mail: xqliu2014@gmail.com

*Contributed equally

Key words: spindle and kinetochore-associated protein 1, glioblastoma, RNA interference, lentivirus two SKA1-SKA2 heterodimers, each of which interacts with a SKA3 homodimer $(1,2,4)$.

Previous studies have demonstrated that the human SKA1 complex plays an important role in human cells. As an important kinetochore component, the human SKA1 complex is involved in the development of kinetochore-microtubule interactions and is essential for proper chromosome segregation (1-3,5-9). SKA1 complex is able to directly associate with microtubules, and has been shown to possess oligomerization-promoting activity, which is provided by the Ramal subunit and catalyzed by the microtubule lattice $(2,5,6)$. Depletion of the SKA1 complex in human cells leads to checkpoint-dependent mitotic arrest with misaligned chromosomes (3). Recently, the role of the SKA1 complex in meiosis was investigated in mouse oocytes (10). In meiosis, the SKA1 complex was not found to be localized at the KT, but on spindle microtubules from the prometaphase I to metaphase II stages, which differed from mitosis. Knockdown of any member of the SKA1 complex results in spindle movement defects and enlarged polar bodies, whereas depletion of the entire SKA1 complex impairs the stability of the anaphase spindle, as well as the extrusion of the first polar body (10).

Glioblastoma possesses two variants, the giant cell glioblastoma and gliosarcoma, and is the most common and aggressive form of brain tumor $(11,12)$. Glioblastoma is associated with high mortality and can strongly invade adjacent normal tissues (13). SKA1 can bind directly to microtubules and plays an important role in human cell division (4). Furthermore, microtubules have been found to be associated with glioblastoma cell invasion (14). Therefore, knockdown of SKA1 expression was hypothesized to impair the proliferation and invasion of glioblastoma cells.

The present study investigated whether SKA1 is expressed in human glioblastoma cells, through the specific knockdown of SKA1 in the A172 and U251 human glioblastoma cell lines using the lentivirus infection method. In addition, the effect of the SKA1 downregulation on cell proliferation, invasion 
and the cell cycle was evaluated. The results of the present study may be helpful in establishing antiproliferative and anti-invasive therapeutic strategies, by targeting SKA1 in human glioblastoma.

\section{Materials and methods}

Cell lines. U251, U87MG, U373 and A172 human glioblastoma and the 293T human kidney cell lines were purchased from the Cell Bank of the Chinese Academy of Sciences (Shanghai, China). All the cell lines were maintained in Dulbecco's modified Eagle's medium (DMEM; Hyclone Laboratories, Inc., Logan, UT, USA) supplemented with $10 \%$ fetal bovine serum (FBS; Hyclone Laboratories, Inc.), $1 \%$ penicillin/streptomycin and L-glutamine (Hyclone Laboratories, Inc.), at $37^{\circ} \mathrm{C}$ in a $5 \% \mathrm{CO}_{2}$ atmosphere. The study was approved by the Ethics Committee of Shanghai Tenth People's Hospital, Tonji University School of Medicine, Shanghai, China.

Construction of small hairpin (sh)RNA-expressing plasmids. The human SKA1 cDNA sequence was obtained from GenBank (no. NM_001039535). According to the obtained cDNA sequence, the following shRNA targeting SKA1 was designed: 5'-CCGGCCTGACACAAAGCTCCTAAATCT CGAGATTTAGGAGCTTTGTGTCAGGTTTTTG-3'. The control shRNA sequence was as follows: 5'-TTCTCCGAACGT GTCACGT-3'. The two shRNAs were inserted into the pFH-L lentiviral vector (Shanghai Hollybio, Shanghai, China) and were termed as pFH-L-shSKA1 or pFH-L-shCon, respectively.

Lentivirus packaging and infection. The 293T cells were transfected with the pFH-L-shSKA1 or pFH-L-shCon plasmid, as well as two helper plasmids, pVSVG-I and pCMV $\Delta$ R8.92 (Shanghai Hollybio), using the Lipofectamine ${ }^{\circledR} 2000$ reagent (Life Technologies, Grand Island, NY, USA). After culturing for $24 \mathrm{~h}$, the virus samples were collected, passed through $0.45-\mu \mathrm{m}$ filters (EMD Millipore, Billerica, MA, USA) to remove the 293T cells and transduced into the A172 or U251 target human glioblastoma cell lines.

Infection efficiency assay. The A172 or U251 cells were seeded in 6-well plates at a density of $5 \times 10^{4}$ or $8 \times 10^{4}$ cells/well, respectively. The lentivirus carrying pFH-L-shSKA1 (Lv-shSKA1) or pFH-L-shCon (Lv-shCon) was used to infect the A172 [multiplicity of infection $(\mathrm{MOI})=8$ ] and $\mathrm{U} 251$ cells $(\mathrm{MOI}=2)$. After culturing for four days, the cells were observed under an Olympus BX50 Brightfield/Fluorescence microscope (Olympus Corporation, Tokyo, Japan). The cells containing the $\mathrm{pFH}-\mathrm{L}$ vector exhibited green fluorescent protein (GFP) fluorescence, whereas no GFP signal was observed in the control cells.

Reverse transcription-quantitative polymerase chain reaction $(R T-q P C R)$. At day 4 after infection, total RNA was isolated from the A172 or U251 cells using TRIzol ${ }^{\circledR}$ reagent (catalog no. 15596-026; Invitrogen Life Technologies, Carlsbad, CA, USA). cDNA was reverse-transcribed using Super Script ${ }^{\circledR}$ II (Invitrogen Life Technologies), according to the manufacturer's instructions. For quantitative thermal cycling, $10 \mu \mathrm{l}$ 2X SYBR ${ }^{\circledR}$ Premix Ex Taq ${ }^{\mathrm{TM}}$ (Bio-Rad Laboratories, Inc.,
Hercules, CA, USA), $0.8 \mu 1$ primers $(2.5 \mu \mathrm{M}), 5 \mu 1 \mathrm{cDNA}$ and $4.2 \mu \mathrm{l}$ double-distilled $\mathrm{H}_{2} \mathrm{O}$ were mixed. A two-step RT-PCR method was performed and the conditions were set as follows: Initial predenaturation at $95^{\circ} \mathrm{C}$ for $1 \mathrm{~min}$, followed by amplification for 40 cycles at $95^{\circ} \mathrm{C}$ for $5 \mathrm{sec}$ and at $60^{\circ} \mathrm{C}$ for $20 \mathrm{sec}$. Fluorescence data were measured at the end of each cycle and the expression levels were calculated using the $2^{-\Delta \Delta C t}$ method. All the RT-qPCR experiments were performed using a Bio-Rad Connect ${ }^{\mathrm{TM}}$ Real-Time PCR platform (Bio-Rad Laboratories, Inc.). The primers used in the RT-qPCR analysis were as follows: SKA1 forward, 5'-TGATGTGCCAGGAAG GTGAC-3', and reverse, 5'-CAAAGGATACAGATGAAC AACAGC-3'; and actin (used as an internal control) forward, 5'-GTGGACATCCGCAAAGAC-3', and reverse, 5'-AAA GGGTGTAACGCAACTA-3'. The primers were purchased from Shanghai Hollybio.

MTT assay. A172 or U251 cells were seeded in 6-well plates at a density of $2.5 \times 10^{2}$ cells/well. The cell proliferation ability was assessed using an MTT assay and acidic isopropanol method, at day 4 after lentiviral infection. Briefly, MTT solution ( $5 \mathrm{mg} / \mathrm{ml}$; Sigma-Aldrich, St. Louis, MO, USA) was added to the cells at a volume of $20 \mu \mathrm{l}$ per well, according to the manufacturer's instructions. After $4 \mathrm{~h}$ of incubation, the entire supernatant was discarded and acidic isopropanol (10\% sodium dodecyl sulfate, $5 \%$ isopropanol and $0.01 \mathrm{M} \mathrm{HCl}$; Sigma-Aldrich) was added at a volume of $100 \mu \mathrm{l}$ per well prior to incubation in a $5 \% \mathrm{CO}_{2}$ atmosphere overnight at $37^{\circ} \mathrm{C}$. The absorption values were measured at $595 \mathrm{~nm}$ [optical density (OD)595] using an Epoch Microplate Spectrophotometer (Biotek Instruments, Inc., Winooski, VT, USA).

Flow cytometric analysis. The cell cycle distribution was analyzed by flow cytometry assay. In brief, on day 4 after infection, the A172 or U251 cells were seeded into 6-cm dishes at a density of $3 \times 10^{4}$ or $5 \times 10^{4}$ cells/dish, respectively, and cultured for $40 \mathrm{~h}$. Then cells were collected, washed by pre-cooled phosphate-buffered saline (Sigma-Aldrich) and fixed using $75 \%$ ethanol overnight at $4^{\circ} \mathrm{C}$. Cells were resuspended in $20 \mathrm{mg} / \mathrm{ml}$ propidium iodide solution (Sigma-Aldrich). The samples were analyzed by a FACS Calibur flow cytometer (BD Biosciences, San Jose, CA, USA).

Cell invasion assay. Cell motility and migration were assessed using Transwell chambers (8.0- $\mu \mathrm{m}$ pores; 24 -wells; Corning Incorporated, Corning, NY, USA). The two cell lines were synchronized in three different groups (Con, Lv-shCon and Lv-shSKA1) in triplicate. On day 3 after infection, the A172 or U251 cells were seeded in the upper chamber, at a density of $3 \times 10^{4}$ or $5 \times 10^{4}$ cells/well, respectively. DMEM supplemented with $10 \%$ FBS was added in the lower chamber. Following incubation for one day, the migrated cells in the lower chamber were fixed with methanol and stained crystal violet (Beyotime Institute of Biotechnology, Shanghai, China). The samples were visualized and images were captured on a florescence microscope (Olympus Corp.). The absorption values were measured at $570 \mathrm{~nm}$.

Statistics analysis. The data were analyzed using unpaired two-tailed Student's t test and are presented as the 
A

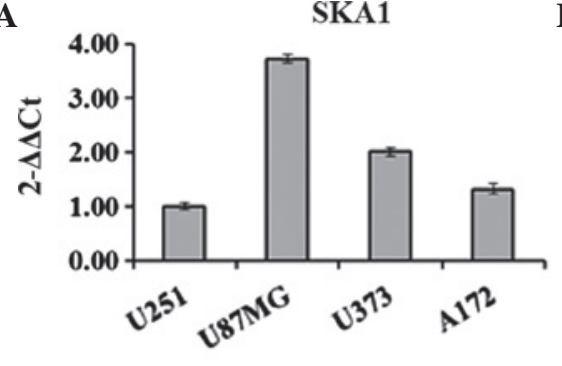

B

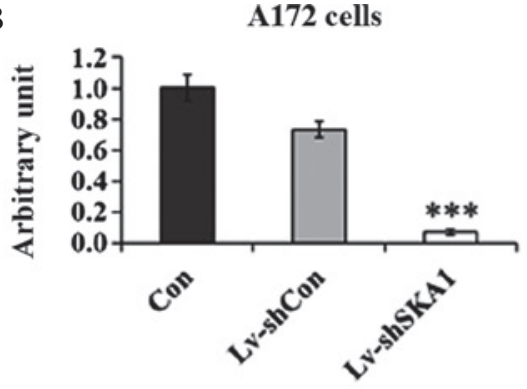

C

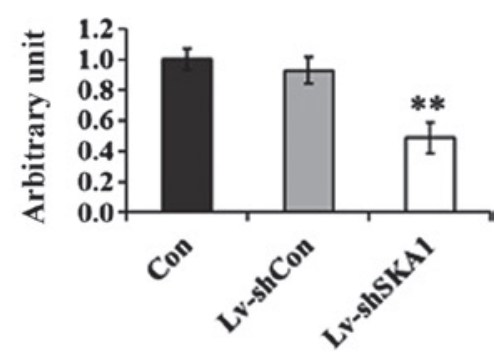

D

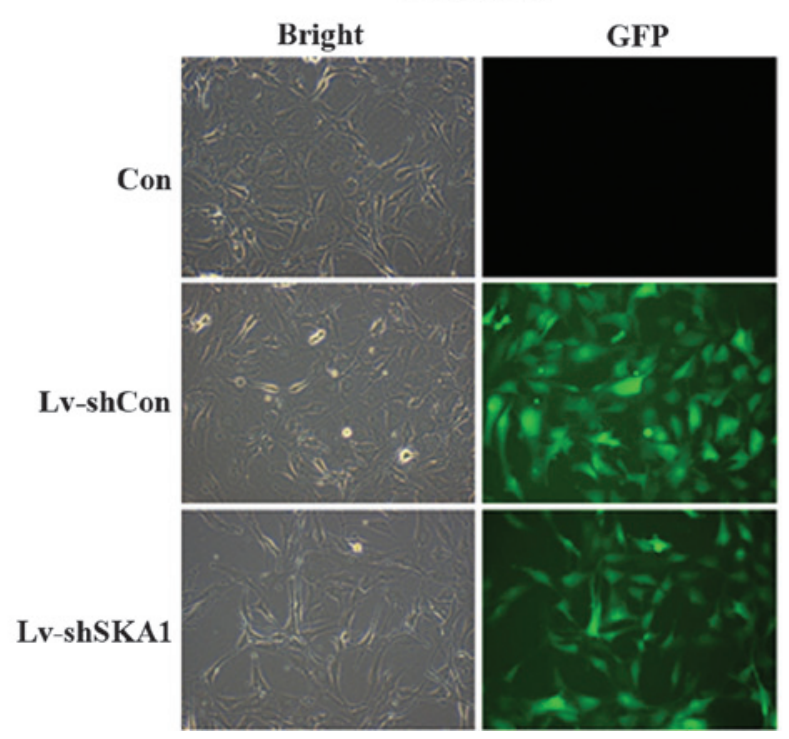

U251 cells

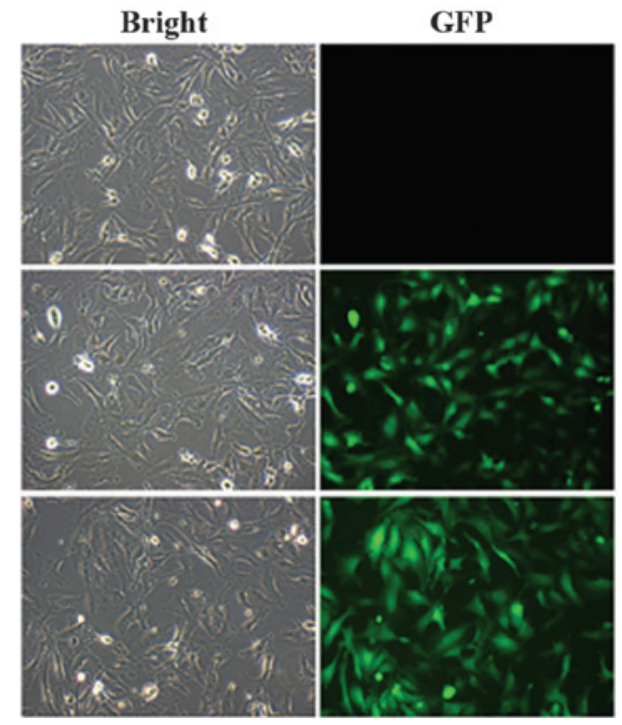

Figure 1. Expression levels of SKA1 are shown in the U251, U87MG, U373 and A172 human glioblastoma cell lines, determined by reverse transcription-quantitative polymerase chain reaction (RT-qPCR) that was performed using cell line cDNA sequences, with actin as the control. (A) The effect of knockdown of SKA1 expression on (B) A172 and (C) U251 cells infected with Lv-shCon or Lv-shSKA1, determined by RT-qPCR that was performed using cDNA from the control and transfected cells. The data are presented as the mean \pm standard deviation. ${ }^{* *} \mathrm{P}<0.05$ and ${ }^{* * * *} \mathrm{P}<0.001$, vs. Lv-shCon group. (D) Green fluorescence protein assay demonstrating the infection efficiency of lentivirus on the A172 and U251 cells (magnification, x100). SKA1, spindle and kinetochore-associated protein 1; Con, control cells; Lv-shCon, cells transfected with control small hairpin (sh)RNA and lentivirus vector; Lv-shSKA1, cells transfected with SKA1-targeting shRNA and lentivirus vector.

mean \pm standard deviation. Statistical analysis was performed using Prism 5 for Windows software (GraphPad Software, San Diego, CA, USA). $\mathrm{P}<0.05$ was considered to indicate a statistically significant difference.

\section{Results}

Expression levels of SKAl in human glioblastoma cell lines. RT-qPCR was performed to determine the expression levels of SKA1 in the human glioblastoma cell lines. As shown in Fig. 1A, mRNA expression of SKA1 was detected in all the selected human glioblastoma cell lines. The transcription level of SKA1 was higher in the U87MG cells compared with other cells. The two cell lines with moderate SKA1 expression levels, A172 and U251, were used in subsequent experiments.

Knockdown of SKA1 expression by lentivirus infection in human glioblastoma cells. In order to investigate the role of SKA1, an shRNA sequence targeting SKA1 was designed to specifically knockdown SKA1 expression in human glioblastoma cells. At day 4 after infection with Lv-shSKA1, the mRNA expression levels of SKA1 were found to be significantly downregulated in the A172 and U251 cells, when compared with the control and Lv-shCon-infected cells (Fig. 1B and C). The knockdown efficiency in the A172 cells was $90.2 \%$, which was higher compared with the U251 cells (47.4\%). Since a GFP marker is present in the pFL-H vector, the cells were observed under a microscope to further calculate the infection efficiency of the lentivirus into the human glioblastoma cells. Most of the cells exhibited a GFP-positive signal following infection for four days (Fig. 1D). These results indicate that SKA1 expression was efficiently knocked-down in the human glioblastoma cells.

Knockdown of SKAl expression inhibits proliferation in human glioblastoma cells. SKA1 has been reported to be associated with cell mitosis and division. Therefore, the present study aimed to determine the effect of SKA1 on the regulation of cell proliferation. As shown in Fig. 2, the results of the MTT assay indicated that cell proliferation rates were significantly decreased following knockdown of SKA1 expression in the A172 and U251 cells, at days 4 and 5 after infection (Fig. 2). On day 5, the OD595 value of the A172 cells infected with Lv-shSKA1 was found to be $\sim 50 \%$ lower compared with the 
A

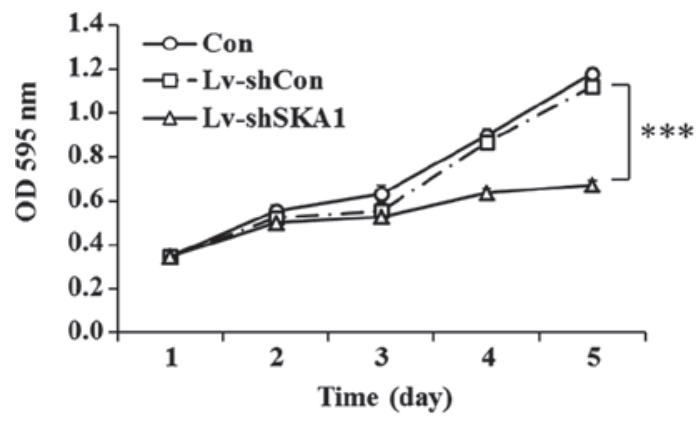

B

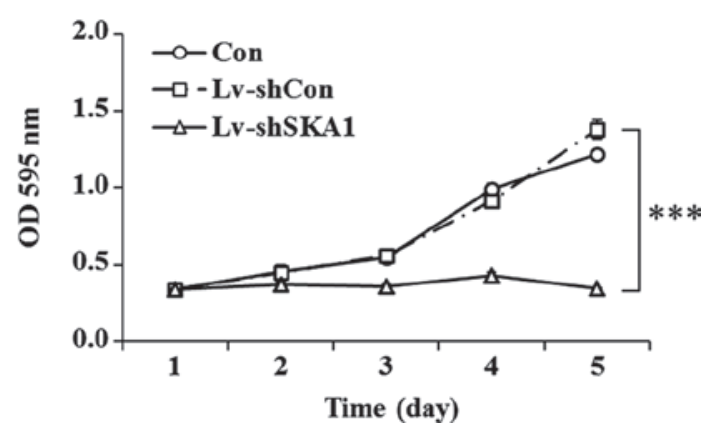

Figure 2. Knockdown of SKA1 inhibited the cell proliferation rates of (A) A172 and (B) U251 human glioblastoma cells, determined by MTT assay. ${ }^{* * *} \mathrm{P}<0.001$. SKA1, spindle and kinetochore-associated protein 1; OD, optical density; Con, control cells; Lv-shCon, cells transfected with control small hairpin (sh)RNA and lentivirus vector; Lv-shSKA1, cells transfected with SKA1-targeting shRNA and lentivirus vector.
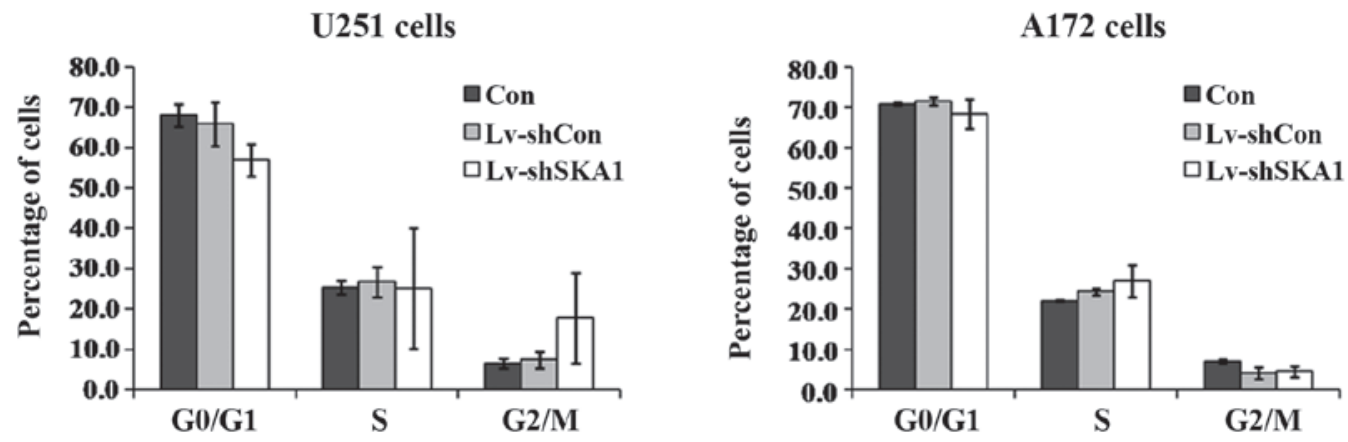

Figure 3. Downregulation of SKA1 expression did not affect the cell cycle of the A172 and U251 human glioblastoma cells. The data are presented as the mean \pm standard deviation. SKA1, spindle and kinetochore-associated protein 1; Con, control cells; Lv-shCon, cells transfected with control small hairpin (sh)RNA and lentivirus vector; Lv-shSKA1, cells transfected with SKA1-targeting shRNA and lentivirus vector.

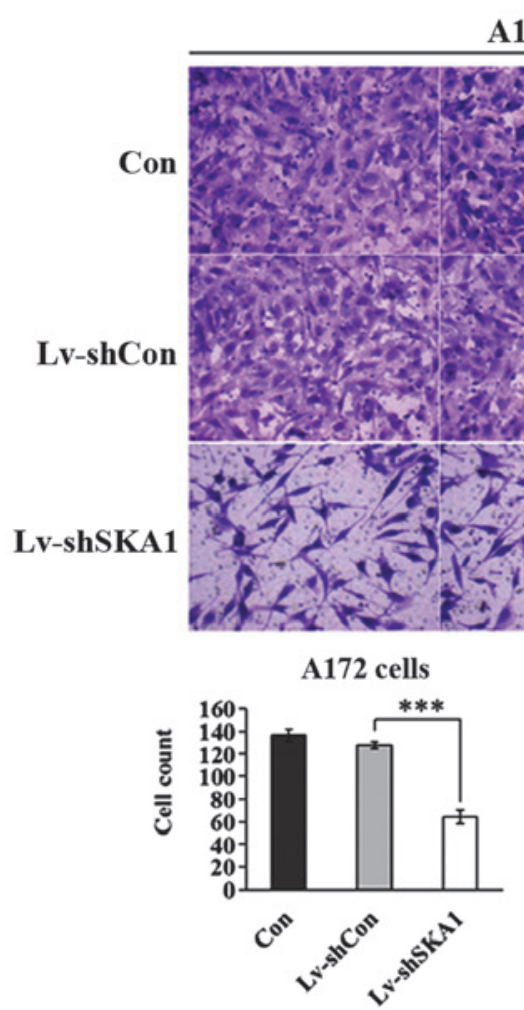

A172 cells

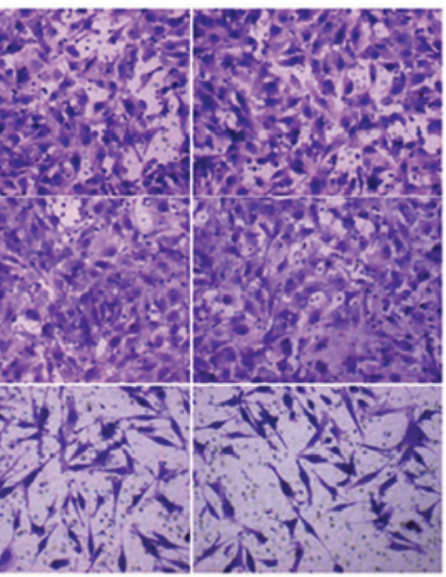

A172 cells

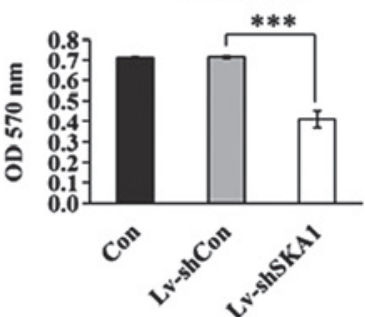

U251 cells

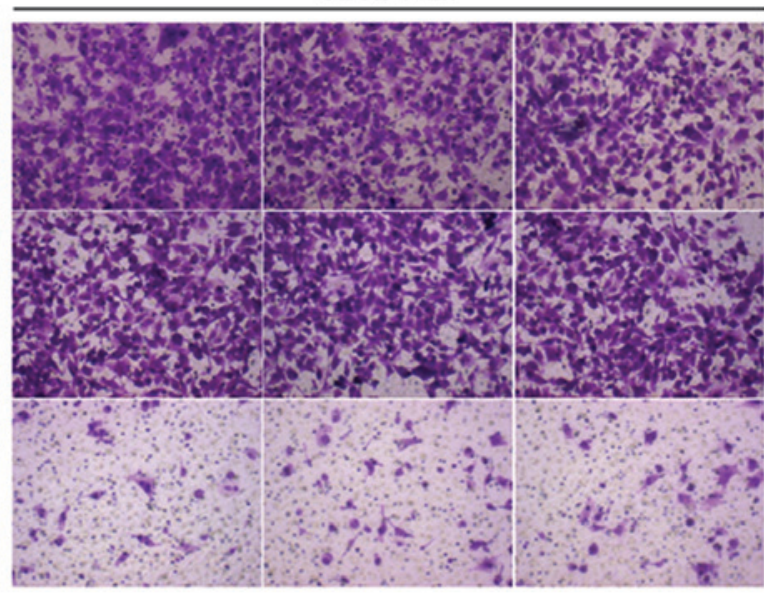

U251 cells

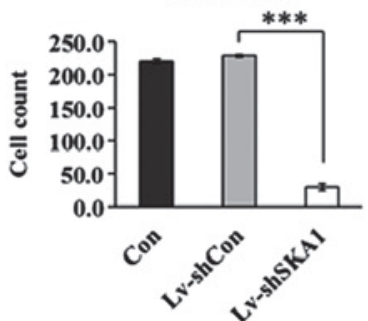

U251 cells

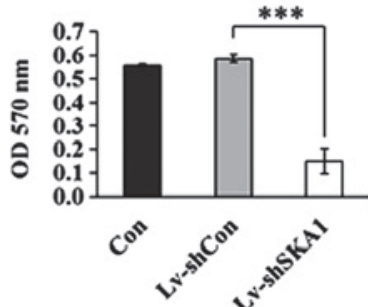

Figure 4. Knockdown of SKA1 expression suppressed cell invasion in A172 and U251 human glioblastoma cells, analyzed by transwell invasion assay. The cells were stained with crystal violet and the absorption values were measured at $570 \mathrm{~nm}$ (magnification, $\mathrm{x} 100$ ). The data are presented as the mean \pm standard deviation. ${ }^{* * *} \mathrm{P}<0.001$. SKA1, spindle and kinetochore-associated protein 1; OD, optical density; Con, control cells; Lv-shCon, cells transfected with control small hairpin (sh)RNA and lentivirus vector; Lv-shSKA1, cells transfected with SKA1-targeting shRNA and lentivirus vector. 
control cells. By contrast, the OD595 value of the U251 cells was $\sim 75 \%$ lower compared with the control cells. These results indicate that downregulation of SKA1 may inhibit proliferation of human glioblastoma cells.

Downregulation of SKA1 does not affect the cell cycle progression in human glioblastoma cells. To determine whether SKA1 regulates cell proliferation through cell cycle control, flow cytometric analysis was performed to calculate the number of cells in the different stages of the cell cycle. No statistically significant variations were identified in the number of cells in the G0/G1, S and G2/M stages between the control, Lv-shCon or Lv-shSKA1-infected A172 and U251 cells (Fig. 3). The results indicate that downregulation of SKA1 does not influence the cell cycle progression in human glioblastoma cells.

Knockdown of SKA1 expression suppresses cell invasion in human glioblastoma cells. Invasion into the surrounding tissues is important for the dissemination of cancer. To determine the role of SKA1 in the invasion of human glioblastoma cells, a transwell invasion assay was performed using A172 and U251 cells. When compared with the control cells, a much lower number of LV-shSKA-infected A172 and U251 cells were found to penetrate the membrane at $24 \mathrm{~h}$ after seeding (Fig. 4). In the control A172 and U251 cells, the number of cells that migrated into the lower chamber were $136.5 \pm 6.0$ and $219.7 \pm 3.8$, respectively. In the Lv-shSKA1-infected A172 and U521 cells, $64.3 \pm 6.2$ and $28.7 \pm 5.2$ cells migrated, respectively. By contrast, the number of migratory cells in the Lv-shCon-infected A172 and U521 cells was $127.9 \pm 3.2$ and $228.9 \pm 2.0$, respectively. These findings were in accordance with the obtained OD570 values. The results indicate that downregulation of SKA1 may suppress cell invasion in human glioblastoma cells.

\section{Discussion}

Human glioblastoma has been identified as the most frequent and malignant tumor of the central nervous system in adults $(11,15,16)$. A number of factors in human glioblastoma result in poor prognosis of $\sim 5$ years (17). High proliferation rates, extensive invasion and dissemination into adjacent non-neoplastic brain tissue prevent efficient surgical resection of the tumor (18). Previous studies have identified various proteins involved in tumor proliferation and invasion, including growth factors, adhesion molecules and proteases, as well as extracellular matrix and cell cycle components $(19,20)$. To the best of our knowledge, the present study demonstrated for the first time that downregulation of SKA1, an important component of the human $\mathrm{KT}$, can inhibit proliferation and invasion of human glioblastoma cells.

SKA1 has been identified as a key component of the KT-microtubule-associated complex, which is involved in cell division $(1-3,5,6)$. Previous studies have demonstrated that RNA interference targeting SKA1 results in the loss of the SKA1 complex from the KT, impairing the KT-microtubule interface and compromising proper chromosome segregation, without disruption of the overall KT structure $(1,2)$. The results of the present study revealed that knockdown of SKA1 expression significantly inhibited cell proliferation, which is in accordance with the findings of previous studies.
Microtubules and certain microtubule-associated proteins have been shown to be involved in the motility and proliferation of glioblastoma cells (21-24). Microtubules serve as targets of certain widely-used conventional anticancer tubulin-binding agents (21). In vitro studies have revealed that the SKA1 complex possesses two separate biochemical activities, the direct microtubule binding through SKA1 and the microtubule-stimulated oligomerization through the Ramal component $(2,4)$, which facilitate the movement of microspheres along the depolymerized microtubule. Downregulation of SKA1 may disrupt the microtubule structure, hence inhibiting cell proliferation and invasion. These results indicate that SKA1 may be a potential target in the treatment of human glioblastoma.

In conclusion, the present study demonstrated that SKA1 is expressed in human glioblastoma cells. Lentivirus infection was found to efficiently knockdown SKA1 expression in the A172 and U251 human glioblastoma cell lines. Furthermore, downregulation of SKA1 inhibited cell proliferation and invasion, without affecting the cell cycle of human glioblastoma cells. These results indicate that SKA1 may affect glioblastoma proliferation and invasion. The present study may be helpful in the development of an antiproliferative and anti-invasive treatment of human glioblastoma by targeting SKA1.

\section{Acknowledgements}

This study was supported by grants from the National Science Foundation of China (nos. 81100673, 81202550, 81370457 and 81350110521), the National Science Foundation 973 program (no. 2011CB965100), China Postdoctoral Science Foundation (no. 2013M541548), 2013 Young Talents Cultivation Program of Tongji University (no. 2013KJ057), STCSM (no. 12ZR1423400), and the Science and Technology Agency of Zhejiang Province (no. 2012C23109).

\section{References}

1. Hanisch A, Silljé HH and Nigg EA: Timely anaphase onset requires a novel spindle and kinetochore complex comprising Ska1 and Ska2. EMBO J 25: 5504-5515, 2006.

2. Welburn JP, Grishchuk EL, Backer CB, Wilson-Kubalek EM, Yates JR III and Cheeseman IM: The human kinetochore Ska1 complex facilitates microtubule depolymerization-coupled motility. Dev Cell 16: 374-385, 2009.

3. Guimaraes GJ and Deluca JG: Connecting with Ska, a key complex at the kinetochore-microtubule interface. EMBO J 28: 1375-1377, 2009

4. Jeyaprakash AA, Santamaria A, Jayachandran U, et al: Structural and functional organization of the Ska complex, a key component of the kinetochore-microtubule interface. Mol Cell 46: 274-286, 2012.

5. Gaitanos TN, Santamaria A, Jeyaprakash AA, Wang B, Conti E and Nigg EA: Stable kinetochore-microtubule interactions depend on the Ska complex and its new component Ska3/C13Orf3. EMBO J 28: 1442-1452, 2009.

6. Theis M, Slabicki M, Junqueira M, et al: Comparative profiling identifies C13orf3 as a component of the Ska complex required for mammalian cell division. EMBO J 28: 1453-1465, 2009.

7. Schmidt JC, Arthanari H, Boeszoermenyi A, et al: The kinetochore-bound Skal complex tracks depolymerizing microtubules and binds to curved protofilaments. Dev Cell 23: 968-980, 2012.

8. Zhang G, Kelstrup CD, Hu XW, et al: The Ndc80 internal loop is required for recruitment of the Ska complex to establish end-on microtubule attachment to kinetochores. J Cell Sci 125: 3243-3253, 2012

9. Daum JR, Wren JD, Daniel JJ, et al: Ska3 is required for spindle checkpoint silencing and the maintenance of chromosome cohesion in mitosis. Curr Biol 19: 1467-1472, 2009. 
10. Zhang QH, Qi ST, Wang ZB, et al: Localization and function of the Ska complex during mouse oocyte meiotic maturation. Cell Cycle 11: 909-916, 2012.

11. Anton K, Baehring JM and Mayer T: Glioblastoma multiforme: overview of current treatment and future perspectives. Hematol Oncol Clin North Am 26: 825-853, 2012.

12. Pool JL: The management of recurrent gliomas. Clin Neurosurg 15: 265-287, 1968.

13. Yoshida T, Matsuda Y, Naito Z and Ishiwata T: CD44 in human glioma correlates with histopathological grade and cell migration. Pathol Int 62: 463-470, 2012.

14. Pagano A, Honoré S, Mohan R, et al: Epothilone B inhibits migration of glioblastoma cells by inducing microtubule catastrophes and affecting EB1 accumulation at microtubule plus ends. Biochem Pharmacol 84: 432-443, 2012.

15. Rostomily RC, Spence AM, Duong D, McCormick K, Bland M and Berger MS: Multimodality management of recurrent adult malignant gliomas: results of a phase II multiagent chemotherapy study and analysis of cytoreductive surgery. Neurosurgery 35: 378-388, 1994.

16. Hou LC, Veeravagu A, Hsu AR and Tse VC: Recurrent glioblastoma multiforme: a review of natural history and management options. Neurosurg Focus 20: E5, 2006.

17. Liu BL, Cheng JX, Zhang $X$ and Zhang W: Controversies concerning the application of brachytherapy in central nervous system tumors. J Cancer Res Clin Oncol 136: 173-185, 2010.
18. Grah JJ, Katalinic D, Stern-Padovan R, et al: Leptomeningeal and intramedullary metastases of glioblastoma multiforme in a patient reoperated during adjuvant radiochemotherapy. World J Surg Oncol 11: 55, 2013.

19. Tysnes BB and Mahesparan R: Biological mechanisms of glioma invasion and potential therapeutic targets. J Neurooncol 53: 129-147, 2001.

20. Kwiatkowska A and Symons M: Signaling determinants of glioma cell invasion. Adv Exp Med Biol 986: 121-141, 2013.

21. Katsetos CD and Dráber P: Tubulins as therapeutic targets in cancer: from bench to bedside. Curr Pharm Des 18: 2778-2792, 2012

22. Katsetos CD, Draber P and Kavallaris M: Targeting $\beta I I I-t u b u l i n$ in glioblastoma multiforme: from cell biology and histopathology to cancer therapeutics. Anticancer Agents Med Chem 11: 719-728, 2011.

23. Katsetos CD, Dráberová E, Legido A, Dumontet C and Dráber P: Tubulin targets in the pathobiology and therapy of glioblastoma multiforme. I. Class III beta-tubulin. J Cell Physiol 221: 505-513, 2009.

24. Katsetos CD, Dráberová E, Legido A and Dráber P: Tubulin targets in the pathobiology and therapy of glioblastoma multiforme. II. gamma-tubulin. J Cell Physiol 221: 514-520, 2009. 